\title{
A Hybrid Particle Swarm Optimization with Affine Transformation Approach for Cloud Free Multi-Temporal Image Registration
}

\author{
Swarna Priya RM*', S Prabu+, V S Dharun ${ }^{+}$ \\ *Research Scholar, School of Computer Engineering, VIT University, Vellore, Tamil Nadu 632014. \\ ${ }^{+}$School of Computer Engineering, VIT University, Vellore, Tamil Nadu 632014. \\ ${ }^{+}$Department of Biomedical Engineering, Noorul Islam University, Kumaracoil, Tamil Nadu
}

Received 1st Jul 2015; accepted 7th Jan 2016

\begin{abstract}
Image Registration (IR) is the process of transformation of different data into the coordinate system and provides the geometric alignment of two images used in the computer vision, medical imaging and remote sensing applications. An image registration is an important stage in multi-temporal image processing since, the recovery of information from cloud shadow is difficult. Traditionally, the Demons, Combined Registration and Segmentation (CRS) approach, Markov Random Field (MRF) and Mutual Information (MI) based approaches offers more computational complexity, minimum edge preservation measure $(\mathrm{QAB} / \mathrm{F})$ during image registration process. To maximize the quality of edge preservation measure and MI with minimum computational time, this paper proposes hybrid Particle Swarm Optimization (PSO)-Affine Transformation (AT) technique for an image registration. An enhanced registration process and the cloud removal technique are proposed for quality improvement of an image. Initially, Gaussian filtering in the preprocessing stage removes the noises present in an image. The proposed PSO extracts the matching points between the reference image and target image in the multitemporal image dataset. Then, the AT on extracted matching points provides the specific feature points from main features. Finally, the Relevance Vector Machine (RVM) classification forms the cluster of specific feature points. The extracted feature points from PSO-AT maximize the quality of edge preservation and MI with efficient cloud removal. The comparative analysis with the traditional methods of Control Point -Least Square (CP-LS), MultiFocus Image Fusion (MFIF) and Discrete Wavelet Transform (DWT) on the parameters of QAB/F and MI shows the effectiveness of proposed PSO-AT.
\end{abstract}

Keywords -Affine Transformation (AT), Control Point-Least Square (CP-LS), Combined Registration Segmentation (CRS), Edge Detection, Image Registration, Markov Random Field (MRF), Multi-Temporal Image (MTI), Multi-Temporal Image Processing (MTIP), Mutual Information (MI), Particle Swarm Optimization (PSO), Relevance Vector Machine (RVM), Remote Sensing (RS) and Synthetic Aperture Radar (SAR).

\section{Introduction}

Environmental monitoring and Change Detection (CD) are evolving research strategies to get prior information about changes in weather patterns. $\mathrm{CD}$ is the process of extracting, analyzing, and defining change information from Remote Sensing (RS) images. Usually, CD refers to discerning the changed areas on two registered RS images at two different times.

Correspondence to: rm.swarna@,gmail.com

Recommended for acceptance by Filiberto Pla

http://dx.doi.org/10.5565/rev/elcvia. 775

ELCVIA ISSN: $1577-5097$

Published by Computer Vision Center / Universitat Autonoma de Barcelona, Barcelona, Spain 
The acquisition of earth information without any physical contact can be done via RS. The increasing use of registration and classification schemes makes the Multi-Temporal Image Processing (MTIP) effective in RS applications such as surveillance, spatial health data analysis, and map updating. Registration is the initial stage in MTIP, and it relies on knowledge of intensities and features of an image, and it can be classified twofold: intensity-based (or area-based) and feature-based methods. Area-Based (AB) methods are more sensitive to intensity changes and distortions in satellite images that lead to poor registration performance. Hence, Feature-Based (FB) methods are used for RS applications. The extracted features from FB methods calculate the geometric correspondence between images from features like edges, contours, and regions regarded as the tie-points of an image. The matching of tie points during an image registration introduces the CD error.

An adaptive decision based on a threshold is used in Image Processing (IP) to minimize the CD error obtained with unsupervised $C D$. The unsupervised $C D$ can be categorized in the spatial domain and the transform domain. The normalization of Multi-Temporal Images (MTIs), i.e. registration, is done in the spatial domain. The MTIs are taken from the geographical areas in different time using mathematical operators like the single band or vector differencing. The Change Vector Analysis (CVA) algorithm generates the image difference by comparison of their vector magnitudes. Finally, the single hypothesis testing threshold scheme detects the changes in MTIs effectively. The spatial domain is proper for optical and radar images obtained with the prescribed quality and it is adequate for the reception of images with different qualities. The signal's energy is compacted thanks to the retention of a few coefficients in the transform domain. Initially, the input images are converted to a common transform domain (either in the wavelet or the pyramid domain). Subsequently, fusion is applied to the transformed coefficients to get a fused image suitable to RS image analysis.

The cloud cover and cloud shadow are the two main noise sources in RS image analysis. Many RS applications such as target detection and earth observation have difficulties when it comes to recovering information from contaminated clouds and shadows. The edge preservation measure $\mathrm{QAB} / \mathrm{F}$ and the Mutual Information (MI) are useful criteria for effective MTIP. Thus, filter- and index-based approaches can recover missing Quantitative RS (QRS) information that requires more computational time. Well-established methods like Combined Registration and Segmentation (CRS), Markov Random Field (MRF), Control Point-Least Square (CP-LS) and the MI suffer when handling large computational complexities, minimum edge preservation measures and adequate MI. Hence, a suitable technique is required to remove cloud shadow in RS applications. In this paper, an enhanced cloud removal technique using Affine Transformation (AT) is proposed to reduce the computational complexity and running time as the number of pixels increases while keeping maximum $\mathrm{QAB} / \mathrm{F}$. The novelty and the technical contributions of the proposed method are

- Acquisition of RS data more than one time;

- Accurate identification of the significant information about world changes and the relationship between environmental and human activities;

- The use of Particle Swarm Optimization (PSO) and Affine Transformation (AT) for image registration and cluster formation; and

- The problem of finding dissimilarities that hint $\mathrm{CD}$ in a feature space is recast as a multi-objective function optimization.

This paper has been organized into five sections. The Section II presents a description about the earlier research works relevant to MTIP. Section III involves the detailed description about the proposed PSO-AT cloud free MTI registration process. Section IV presents the performance analysis of the proposed method. The conclusion and future work are discussed in Section V.

\section{Related Work}

This section examines works related to the MTIP and how registration mechanisms enhanced the performance of RS. An analysis of high resolution images obtained from satellite required for target detection. But, the raise of false alarms degraded the performance of image processing. Kim et al. [1] analyzed the TerraSAR-X and WorldView-2 high resolution images. They used Constant False Alarm Rate (CFAR) in order to reduce the false alarms. The unpredictable changes in weather patterns from the high resolution images to be investigated from environmental monitoring. The raise up of unsupervised and supervised change 
detection methods effectively predict the changes in weather patterns and high resolution images. Celik et al. [2] presented an unsupervised image change detection method for the satellite images, Synthetic Aperture Radar (SAR) images and optical images. Gongo et al. [3] extended the unsupervised change detection with the help of novel fuzzy clustering algorithm. The results of the improved fuzzy algorithm showed the less error over the existing works. Brooks et al. [4] integrated residuals from the harmonic regression with the statistical quality control charts. These charts has the ability to detect the changes in weather by deforestation and degradation. The Urban Density (UD) from multi-spectral images and the integration of SAR and LiDAR were difficult in chart based analysis. Unsupervised methods provided the binary change detection approach where the changes are only highlighted. Alternatively, the evolution of supervised change detection methods highlighted the kind of changes in the land cover regions. Demir et al. [5] presented a novel iterative-based active learning method for detection of changes in land coverage regions in the remote sensing images.

In RS, the images taken from different viewpoints with different sensors required overlapping for alignment of geometric termed as image registration. Zitova et al. [6] reviewed the classical registration methods based on area, feature. They categorized the registration methods according to operational stages such as feature detection, mapping, matching, transformation and resampling. The related problems and the future direction to the development of an image registration also presented. The correspondence between near and away locations used to reduce the consistency errors. Intensity-Based approaches in research works contributed towards the assurance of correspondence. Johnson et al. [7] presented the two algorithms for image registration with one among is founded on matching of landmarks and the other is based on matching of landmarks and intensities. The joint estimation of forward and reverse transformation between two images performed for consistency error detection. The similarity measure of aligning the image from two different modalities defined by simple correlation coefficient. Due to the presence of outlier in the images, the estimation of correlation coefficient leads to biased registration. Kim et al. [8] used the robust correlation co-efficient to reduce the influence of outliers. The comparative analysis with the MI-Based method useful for image surgical applications. Some of the applications required the pixel registration, and some of them required the sub-pixel registration for large convergence range. Transform-based approaches raised in image analysis to achieve the subpixel accurate registration. Averbuch and Keller [9] derived the Fast Fourier Transform (FFT) based image registration for estimation of large translations with maximum sub-pixels accuracy. Local distortions were occurred due to the high data volume. Stone et al. [10] presented the discrete transform approach for enhancement of subpixel registration accuracy. They proposed an algorithm to detect the unreliable transform coefficients and removed by employing of shift-estimation computation.

The construction of high realistic 3D geometric models require automatic registration. The correspondence formation using positional distance vectors involves the feature based registration. Sharp et al. [11] investigated the utilization of Euclidean invariant features for generalization of closest point feature based registration. Stamos and Leordeanu [12] utilized the range segmentation and feature extraction algorithms for effective registration of complex scans. The terrestrial images with 3D approaches contained high texture information. Hence, change detection in terrestrial images suffered from various problems namely, illumination changes, and distortions and pose regularization. Qin et al. [13] combined the Mobile Laser Scanning point clouds and terrestrial images for change detection at street level leads to pose regularization problems. Kroeger et al. [14] presented the transformation model of image registration based on Structure from Motion (SfM) to solve the problem of pose regularization. The pose errors for all frames of video in 3D models are maximum. The estimation of a 3D space model required the proper merging of all depth images. Ruttle et al. [15] used the Generalized Relaxed Radon Transform to merge all images and they assessed the accuracy of 3D shape reconstruction. Trajectory estimation influences the image registration and tracking to provide the better quality. But, the human-based tracking also presented some problems.

The introduction of optical sensors to the RS IP provided the Hyperspectral Images (HSI) and allowed land cover region characterization. The high-dimensionality nature of pixels in HSI leads to noise occurrence and uncertainty of sources. Camps-Valls et al. [16] focused the HSI classification in scientific disciplines of image processing. Serpico et al. [17] validated the image CD, fusion approach, and feature extraction technique through various experiments. The computational cost for accuracy enhancement was more. Benedek et al. [18] used a new probabilistic method for the integration of building extraction with change detection. The Bayesian model verification was carried out to reduce the computational cost of ensuring accuracy. The deficiency in measure of image registrations affected the performance of image registration. To augment the performance, 
Maximum Likelihood (ML) approaches evolved in the research works. Li et al. [19] combined Control Point (CP) with intensity measures for enhanced ML registration method. The EML registration method maximized the likelihood function for registration parameters estimation. The one alignment method of image from different modalities, time points and subjects was not suitable. Hence, Klein et al. [20] created the tool called Elastix to solve the problems in image registration. They also presented the command-line interface enabled the automated processing of large number of datasets. Rapid development of computational anatomy and absence of justified physical model for inter-subject variations raised the Diffeomorphisms methodology. This involved the optimization procedures to predict the solutions to complex partial differential equations.

Vercauteren et al. [21] proposed the non-parametric diffeomorphism process with the Demons algorithm which is one of the optimization process for exhaustive search space. The free form decompositions by Demons offered the computational efficient solution. Pixel and decomposition based methods in Kennaugh framework were sensitive to noise or mis-registration. Li et al. [22] proposed region-based multi-focus image fusion to segment the images according to spatial frequencies. The edge preservation measure (Q) and the MI between the references images and fused image were measured. In Bayesian-based spectral change detection method, the key dates and periods were missed from the record due to the climatic conditions. Berger et al. [23] took the SAR and air bone Light Detection and Ranging (LiDAR) data to improve the surface reluctance retrievals of the optical data. They also finally demonstrated the usefulness of LiDAR fused with optical data. The observational model for satellite images enhanced with the constraint basis AT.

Joint registration and fusion approaches have been introduced in optimization problems like the formulation by Chen et al. [24] that employed the Expectation-Minimization (EM) algorithm to solve this joint optimization problem. The experiments carried out on several visual, thermal and IR images and also improved fusion performance. The optimization of extracted shape from active contour method provided by tracker. But, the analysis of non-rigid changes was not provided. The coefficients were optimized by using the Gauss-Newton optimization scheme. Mahapatra et al. [25] used the multi-scale graph cut technique on Markov Random Field (MRF) for the spatial regularity model efficiently. The Combined Registration Segmentation (CRS) technique was used to solve the computational complexity. From the study, the preservation of information on the edge and the MI using existing approaches required high computation time and also the processes such as feature extraction and affine transformation for cloud removal have low performance. The enhanced AT-based cloud removal technique for a multi temporal image processing is proposed in this paper.

\section{The PSO-AT Method}

The rationale behind the PSO-AT method is to implement cloud removal in MTIP using AT. The individual processes that make up for the PSO-AT method are depicted in the flow diagram from Fig.1. Initially, MTIs of a same place in various months provided as the input to the system. Hyperspectral 220-band MTIs with different color patterns are used as training images. The reference image and test image data set are given as inputs to the registration process. Gaussian filter is used in image filtering to remove the salt-andpepper noise and speckle noise. After filtering, the extraction of feature points from reference and test image performed by using image rotation. Then, AT is applied to the PSO registration points for cloud removal. The proposed work presents an image registration procedure based on the image edge features between samples present in the given image. According to that edge data, feature points in a binary representation are extracted. Next, the best selection of feature points is achieved by a novel objective function that is part of the PSO optimization algorithm. Then, from that feature point, the pixel matching is extracted and all samples of an image are merged to remove clouds using the novel AT model. 


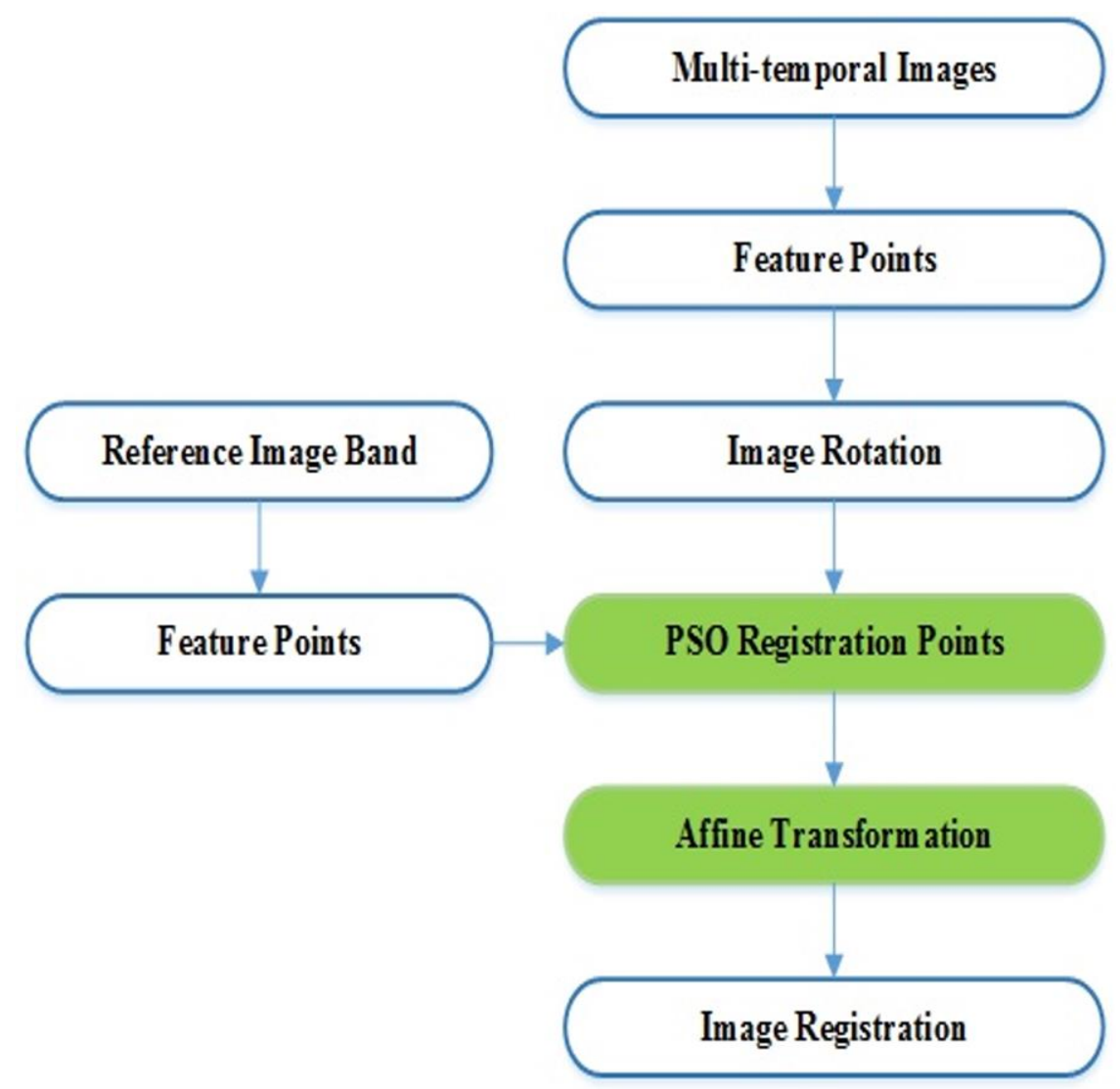

Figure 1: Flow diagram of proposed method.

\subsection{Multi-Temporal Images}

Generally, satellite-based Synthetic Aperture Radar (SAR) images are used in the periodical earth surface monitoring. SAR images investigates the vegetation growth, irrigation and flooding in earth surface. Number of earth observation schemes are involved in the acquisition of MTIs with spatial and temporal resolution. The advantage of satellite-based SAR is the periodical monitoring of earth surface. The investigation about vegetation growth, irrigation and flooding are carried out using SAR image. In SAR process, three images are taken in different times and combined into a single RGB image. The changes in the land usage and land coverage regions are monitored by using MTIP. There are 220 number of bands of images are taken in different times using multi-temporal sensors for an earth surface are used as input in this work.

\subsection{Feature Points Extraction}

Feature extraction is the initial image registration step. Energy compaction is accomplished as the result of compacting the signal's energy into a few transformed coefficients. The database consists of the extracted features, representing the original image matrix from the output of binary image. The MTI from the sensor is recognized as a new image and the features of the new image are compared to the ones from the database to match features. The Euclidean distance between the feature vectors is used as a metric to access feature matching. These matching features are used in the image registration AT process.

\subsection{Image Rotation}

The geometric transform maps the position of a pixel in the input image into the position of an output image by an angle with respect to origin is called image rotation. The rotated coordinates can be obtained by using the following equation:

$x_{2}=\cos (\theta) *\left(x_{1}-x_{0}\right)-\sin (\theta) *\left(y_{1}-y_{0}\right)+x_{0}$, and 
$y_{2}=\sin (\theta) *\left(x_{1}-x_{0}\right)+\cos (\theta) *\left(y_{1}-y_{0}\right)+y_{0}$.

The output locations $\left(x_{2}, y_{2}\right)$ are obtained using rotation of positions $\left(x_{1}, y_{1}\right)$ of an input image with center of origin $\left(x_{0}, y_{0}\right)$ by an angle $\theta$.

The rotation is performed by using two methods. They are the allocation of intensity levels at each integer pixel and the calculation of the intensity level on the basis of $n$ nearest integer values, where $n$ represents the of pixels that correspond to matches between images. The rotation of a new image with respect to the reference image is done by an angle of five degrees as described in Fig. 2 (a) and (b).

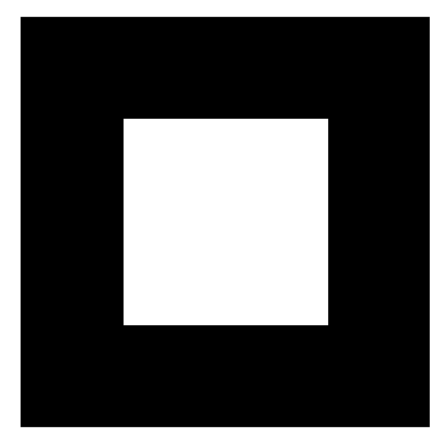

(a)

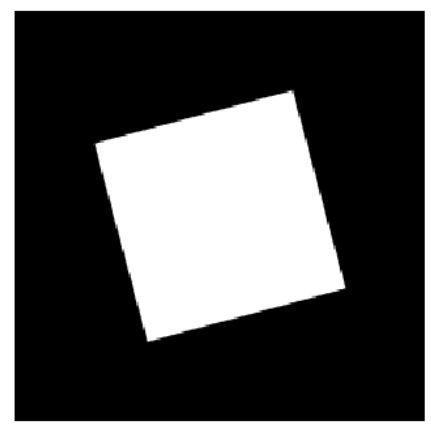

(b)

Figure 2: Image rotation.

\subsection{PSO Registration Points}

PSO is a computational model based on the collaborative behavior of the populations (swarm). Each particle in the population represents the candidate solution. The population of random solutions initiates the system and by updating the generations' optima, populations are searched. Depending upon the information sharing among the population, the search process is split into probabilistic and deterministic methods. The information sharing is done on the global best particle and the swarm reduces the computation time of PSO.

The particle position $\left(X_{i}\right)$ and velocity $\left(V_{i}\right)$ are initialized using following vectors.

$X_{i}=\left(x_{i 1}, x_{i 2}, \ldots \ldots, x_{i D}\right)$

$V_{i}=\left(v_{i 1}, v_{i 2}, \ldots \ldots, v_{i D}\right)$

The fitness function formed from homogeneity and uniformity factors for each particle from position and velocity vectors and compared with the best fitness corresponds to identify pixels in line of curve in swarm. The vectors are updated $\left(V_{i}^{t+1}, X_{i}^{t+1}\right)$ by the identification of best individual using the following equations:

$V_{i}^{t+1}=\omega * V_{i}^{t}+c_{1} * \operatorname{rand}_{1} *\left(P_{i-b e s t}-x_{i}^{t}\right)+c_{2} * \operatorname{rand}_{2} *\left(g_{\text {best }}-x_{i}^{t}\right)$

$X_{i}^{t+1}=\left\{\begin{array}{lr}X_{i}^{t}, & \text { If } V_{i}^{t}>P_{1-\text { best }}^{t} \text { and } G_{\text {best }}^{t} \\ X_{i}^{t}+V_{i}^{t+1}, & \text { else }\end{array}\right\}$

where $i=(1,2 \ldots, N)$

$N$, is the size of the swarm and $P_{i-b e s t}$, is the particle best reached solution. $g_{\text {best }}$, is the global best solution in the swarm. $c_{1}, c_{2}$ are cognitive and social parameters that are bounded between 0 and 2. $\omega$, is the inertia coefficient and instance of time $t$. $\operatorname{rand}_{1}, \operatorname{rand}_{2}$ are two random numbers with uniform distribution $U(0,1)$. 
Equation (3) consists of three components such as inertia, cognitive and social. Inertia weight is the factor used to control the balance between the exploration and exploitation. Cognitive component represents the particle's experience and the social component represents the cooperation among the particles.

Let $p_{i}$ denotes the $i^{\text {th }}$ pixel on the curve $c$. The average homogeneity of the pixels on the curve is denoted by homogeneity factor $\left(h_{c}\right)$ and the pixels on the curve that have the similar values of intensities are denoted by uniformity factor $\left(u_{c}\right) \cdot l_{c}$, is the length of the curve and $I_{p}$, is the intensity of the pixel. Then, the homogeneity and uniformity factor are obtained by using,

$h_{c}=\frac{1}{l_{c}} \sum_{p_{i} \epsilon c} h_{p_{i}}$

$u_{c}=\frac{1}{l_{c}} \sum_{i=1}^{l_{c}-1}\left|I_{P_{i+1}}-I_{p}\right|$

The length of the curve $\left(l_{c}\right)$ is calculated using subsequent expression:

$l_{c}=\sum_{p_{i} \in c}\left\{\begin{array}{c}1 \text { if } p_{i} \text { is odd } \\ \sqrt{2} \text { if } p_{i} \text { is even }\end{array}\right\}$

The objective function $f_{c}$ is formed using the uniformity factor and homogeneity factor $\left(h_{c}\right)$ with the threshold value $(t)$ is given by

$f_{c}=\left\{\begin{array}{ll}-\infty & h_{c}<t \\ \left(h_{c}-u_{c}\right) \times l_{c} & h_{c} \geq t\end{array}\right\}$

The algorithm to find the best fitting curve passing through the pixel contains the sequential measures. Initially, the evaluation of homogeneity factor $\left(h_{c}\right)$, length of curve $\left(l_{c}\right)$, uniformity factor $\left(u_{c}\right)$ and fitness function $\left(f_{c}\right)$ are performed for each pixel. If the calculated fitness value is better than the best fitness value, then the particle is set as new best particle, i.e. the updated fitness function from best fitness point. The neighborhood particle and local best particle are calculated and then the velocity factors and new position are computed until the maximum iterations or stopping criteria is reached. The assigning process of mean value corresponding to the new fitness function which is the required edge containing useful information.

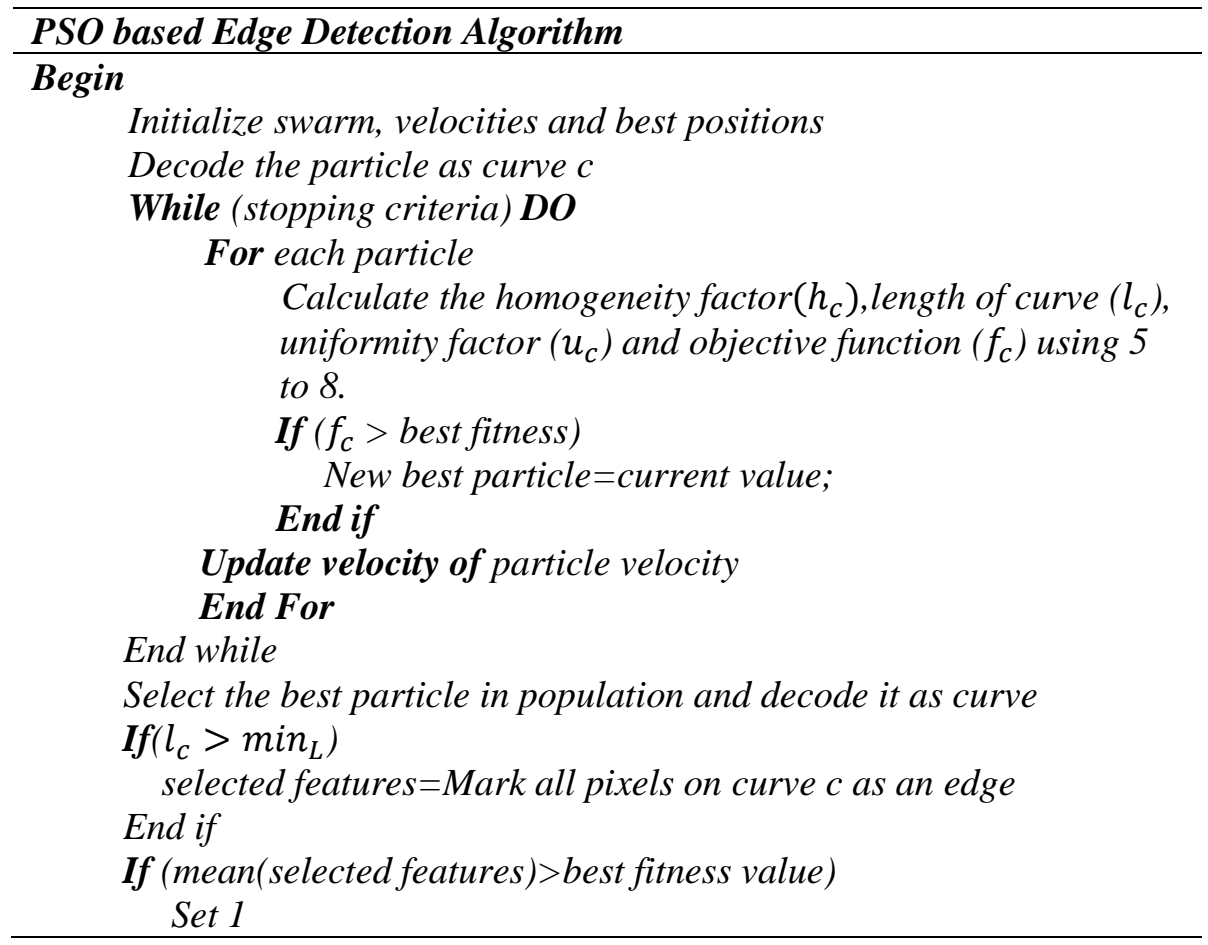




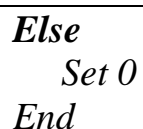

End

The PSO-based registration points provided the best particle corresponds to the features listed. The minimization of feature points required to limit the computation time and complexity. The major difference from traditional method and proposed method is detection of best feature by using a novel model of objective function mentioned in the description of PSO optimization. Moreover, continuous movement of an image affects the positional and velocity vectors which require a great number of iterations. Consequently, the affine transformation applied on the preservation process to extract the main features which reduces the time and complexity.

\subsection{Affine Transformation}

Affine transformation, a matrix multiplication followed by a vector addition which is used to express the rotation, translation and scaling operations. Initially, the points lying on the line is preserved. The nonmovement of images in affine space $\left(r^{n}\right)$ are considered as a special case in transformation. The transformation, which is in the form of matrix multiplication, followed by a vector addition is termed as affine transformation. The affine transformation is used to express the rotation, translation and scaling operations. It preserves the points lying on the line initially. It is the special case of transformation in which the images does not move in affine space and it is denoted by affine space $r^{n}$.

The rotation enlargement transformation, which combines the rotation and expansion which is represented in the following sections.

$\operatorname{Err}_{i, j}=\min _{\arg }\left(\frac{\sum_{i=1}^{N}(\text { vec } 2-v e c 1)}{M}\right)$

vec 1 - Input binary 1.

vec 2 - Input binary 2.

$E r r_{i, j}$ - Difference in vectors

$M$ - Size of vectors

$i, j-$ Row and Column resp.

seeds $=\operatorname{index}(E r r)$

$P t_{\text {indx }}=\bmod \left(\right.$ seeds, size $\left._{\text {vec } 1}\right)$

$i d y=\bmod \left(\right.$ seeds,$\left.b w_{\text {size }}\right)$

$i d x=1+\left(\frac{(\text { seeds-idy) }}{\operatorname{size}(\text { vec } 1)}\right)$

$\alpha=\left\{\left.\left(\tan ^{-1}(b w)\right)\right|_{R}\right\}$

where, $b w$ - Binary pattern of image

$R$ - Region of binary points

$\left[\begin{array}{c}X^{1} \\ Y^{1}\end{array}\right]=S\left[\begin{array}{cc}\cos \alpha & \sin \alpha \\ -\sin \alpha & \cos \alpha\end{array}\right]\left[\begin{array}{c}X-X_{0} \\ Y-Y_{0}\end{array}\right]$

Equations (10) and (11) can be restated as

$$
\begin{aligned}
& \left.X^{1}=(S \cos \alpha) X+(S \sin \alpha) Y-S\left(X_{0} \cos \alpha\right)+Y_{0} \sin \alpha\right) \\
& \left.Y^{1}=(-S \sin \alpha) X+(S \cos \alpha) Y+S\left(X_{0} \sin \alpha\right)-Y_{0} \cos \alpha\right)
\end{aligned}
$$


The equations (10) and (11) can be rewritten as

$$
\begin{aligned}
& X^{1}=A X+B Y-C \\
& Y^{1}=B X+A Y+D
\end{aligned}
$$

The terms A and B represents $S \cos \alpha,-S \sin \alpha$ respectively. The scale factor $(\mathrm{S})$ and the rotation angle $(\alpha)$ are obtained by using

$S=\sqrt{A^{2}+B^{2}}$

Affine space $r^{n}$ represents $\left[\begin{array}{cc}\cos \alpha & \sin \alpha \\ -\sin \alpha & \cos \alpha\end{array}\right]$ and the matrix $P$ denotes $\left[\begin{array}{cc}S \cos \alpha & S \sin \alpha \\ -S \sin \alpha & S \cos \alpha\end{array}\right]$. Then the affine transformation $r^{n}$ is defined as the map $F: r^{n} \rightarrow r^{n}$ of the form $F(P)=A P+Q \forall P, \forall P$.

In equation (18), ' $A$ ' represents the linear transformation and the ' $Q$ ' denotes the translational vectors for transformation. There are two cases depending upon the determinant values of ' $A$ ' (Det $(A))$. If the Det $(A)$ is greater than zero, then the transformation preserving the edges containing information otherwise the edges are not preserved. There are two cases depending upon the determinant values of ' $A$ ' $(\operatorname{Det}(A))$. If the Det $(A)$ is greater than zero, then the transformation preserving the edges containing information otherwise the edges are not preserved. It estimates the variation in pixel intensity as from vecl and vec2 for each frame of the given input image. This represents in the binary form which ' 1 ' indicates the changes in pixel and ' 0 ' indicates there is no any change between them. Then from that binary points, we perform optimization algorithm for selecting best feature point using PSO algorithm. We find distance between them to predict matching between the two binary vectors (vecl and vec2). Finally according to that matched points, we merge the edges of two image samples. After merging the edge points, we find variation in intensity at 'RGB' content of each samples and replace the variation of the pixel which shows the suppress of white cloudy region. Thus we extract the cloud area and suppress it by using the proposed AT model with novel objective of PSO optimization. The affine transformation approaches extracted the required features which reduces the number of iterations required thereby computational time decreased. The approximation of translational vectors based on image centers provided the suitable initial guess reduces the complexity.

\subsection{Image Registration}

The registration of one image with the other image is done by using two methods. The feature points for the reference image and the image for analysis are obtained on the basis of the PSO-based extraction. The detection of the features from different views are done by PSO. By using the AT, the transformation of the matched points between the reference image and analysis image to affine space are performed.

The AT application of PSO-based registration points in fact removes the cloud in an image. From the cloudfree image, the Area Of Interest (AOI) defined outline, shape and the shadow of cloud is extracted after the AT. To reduce the number of ground objects at minimum scale, the interest area relates the cloud. The final cloud-free image is registered using the AT approaches.

\section{Performance Analysis}

This section presents the performance analysis of the proposed cloud removal scheme in MTIP. The proposed scheme used PSO-AT approaches for cloud removal and image registration. The comparative analysis of proposed cloud removal technique with the traditional Combined Registration Segmentation of Markov Random Field model (CRS-MRF) [25], Control-Point Least Square (CP-LS) and MI-based [22, 24] model on the parameters of computational time, edge preservation measure, registration accuracy and registration error rate. The objective function defined in traditional methods formed without suitable initial guess which leads to increase in number of computations and time. Therefore the objective function is made 
by a suitable initial guess reduces the complexity and time considerably. Thereby, the edge preservation measure enhanced. There are 220 Band Hyperspectral Images [26] shown in Fig. 3 (a) and (b) which is acquired by using multi-temporal sensors in various time period is used to validate the PSO-AT methodology.

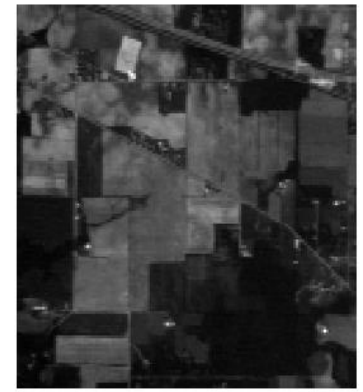

(a)

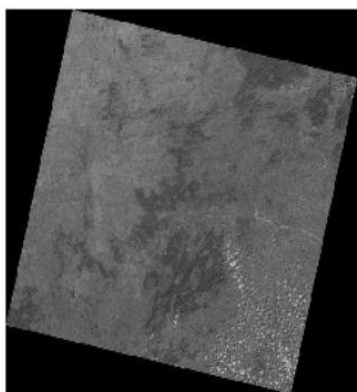

(b)

Figure 3: Multi-Temporal Images.

The input image and the reference image are processed by PSO to extract the registration points are shown in Fig. 4 (a) and Fig. 4 (b). Then the minimization of selected features of reference image and the input image is carried out in Fig.5 (a) and Fig. 5 (b). Input image refers to image with all the bands and Reference image refers to image that we selected with single band. Finally the affine transformation is applied to the image and the cloud-free image is obtained after AT as shown in Fig. 6 (a) and Fig. 6 (b) respectively.

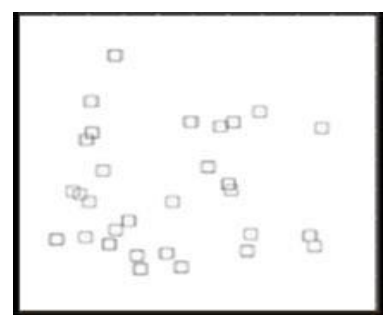

(a)

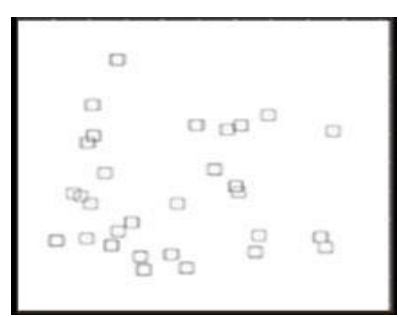

(b)

Figure 4: Feature Extraction.

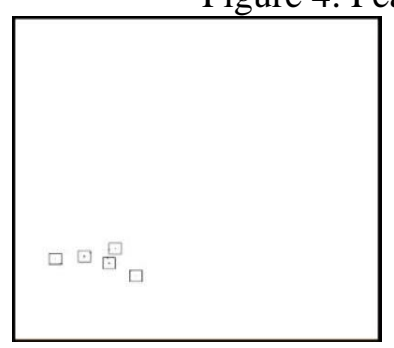

(a)

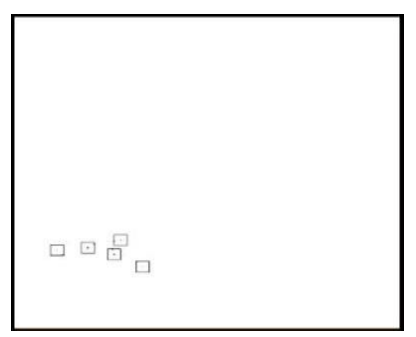

(b)

Figure 5: PSO-based minimized registration points.
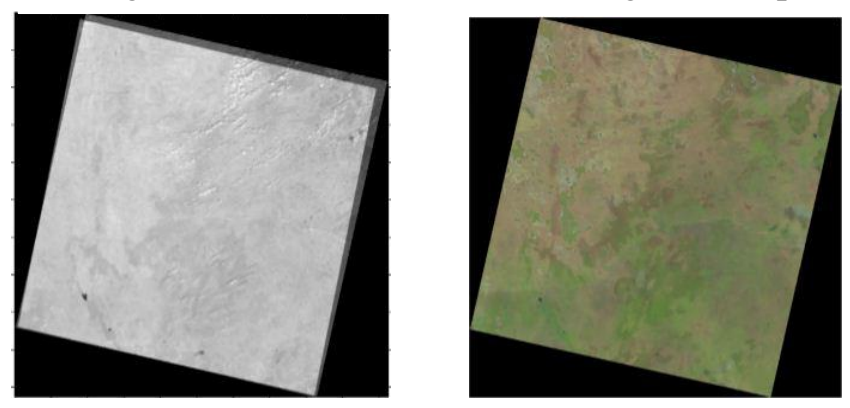

(a)

(b) 
Figure 6: (a) Affine Transform output image, and (b) Cloud-free image.

\subsection{Computational Time}

The number of pixels are changed by increasing the dimension of an image in the ratio of rows to columns as 4:3. The computational time was more for high number of pixels in traditional methods of Demons [21] CRS and MRF [25] than the proposed AT method. Increasing number of pixels effectively reduces the computational time of registration, which also leads to a fast implementation of segmentation of multitemporal images.

Table 1 presents the comparative analysis of the computational times for the proposed PSO-AT method and some traditional methods.

\begin{tabular}{|c|c|c|c|c|}
\hline \multirow{2}{*}{$\begin{array}{c}\text { No. of } \\
\text { Pixels } \\
\left(1 \times 10^{5}\right)\end{array}$} & \multicolumn{4}{|c|}{ Computational Time (secs) } \\
\cline { 2 - 5 } & Demons & CRS & MRF & $\begin{array}{c}\text { Affine } \\
\text { Transformation }\end{array}$ \\
\hline 0.03 & 4 & 10 & 4 & 0.24 \\
\hline 0.035 & 6 & 15 & 6 & 1.68 \\
\hline 0.11 & 20.5 & 30 & 14 & 2.61 \\
\hline 0.16 & 31 & 41 & 18 & 4.53 \\
\hline 0.32 & 75 & 76 & 32 & 6.46 \\
\hline 0.375 & 90.5 & 86 & 40 & 10.41 \\
\hline 0.5 & 123 & 100.5 & 51 & 14.12 \\
\hline 0.8 & 165 & 152 & 72 & 22.85 \\
\hline 0.89 & 198 & 181 & 95 & 25.23 \\
\hline 0.97 & 211 & 197 & 102 & 28.43 \\
\hline 1.5 & 245.5 & 231 & 130 & 42.74 \\
\hline
\end{tabular}

Table 1: Computational Time Vs Pixels

The computational time performance comparison of the AT with the CRS and MRF models is shown in Fig. 7. The graphical representation clearly shows the effectiveness of proposed method in the reduction of computational time.

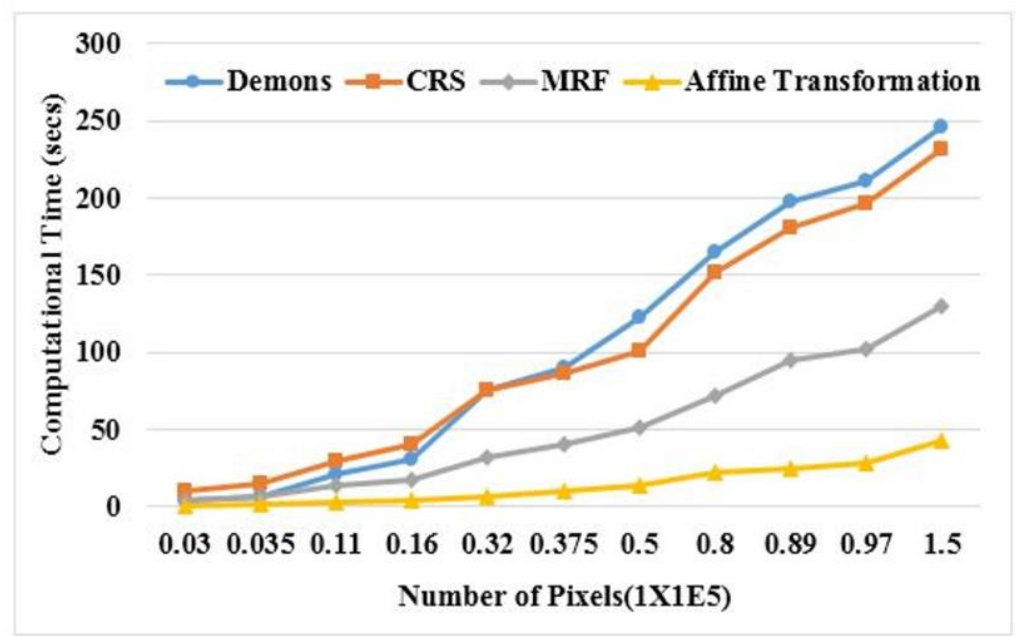

Figure 7: Computational Time vs. No. of Pixels $\left(1 * 10^{5}\right)$

\subsection{Edge Preservation Measure (QAB/F)}

The measure of the edge preservation during the image registration process is obtained using Sobel edge detector. It measure the edge strength $g(y, x)$ and orientation information between the pixels $\alpha(y, x)$. 
Let $\mathrm{A}$ and $\mathrm{B}$ are the two input images converted to the fused image $\mathrm{F}$, then the Sobel edge operator is applied in horizontal and vertical direction $S_{A}^{h}(y, x)$ and $S_{A}^{v}(y, x)$ centered on pixel $p_{A}(y, x)$ are used to find the edge strength and orientation is given by

$$
\begin{aligned}
& g_{A}(y, x)=\sqrt{S_{A}^{h}(y, x)^{2}+S_{A}^{v}(y, x)^{2}} \\
& \alpha_{A}(y, x)=\tan ^{-1}\left(\frac{S_{A}^{v}(y, x)}{S_{A}^{h}(y, x)}\right)
\end{aligned}
$$

where, $\alpha_{A}$ represents the angle of distance for original image and registered image. The relative strength of image A with respect to the fused image $F$. The relative strength of image A with respect to fused image $F$ is calculated as follows:

$$
\begin{aligned}
& \left(G_{y, x}^{A F}, A_{y, x}^{A F}\right)=\left(\left(\frac{g_{A}(y, x)}{g_{F}(y, x)}\right)^{M}, 1-\frac{\left|\alpha_{A}(y, x)-\alpha_{F}(y, x)\right|}{\pi / 2}\right) \\
& \text { where, } M=\left\{\begin{array}{cc}
1 & \text { if } g_{A}(y, x)>g_{F}(y, x) \\
-1 & \text { otherwise }
\end{array}\right\}
\end{aligned}
$$

The sum of edge information preservation values are obtained for the inputs of $Q A / F$ and $Q B / F$ weighted by $w^{A}$ and $w^{B}$ respectively as follows:

$Q A B / F=\frac{\sum_{y, x} Q A / F_{y, x} w_{y, x}^{A}+Q A / F_{y, x} w_{y, x}^{B}}{\sum_{y, x} w_{y, x}^{A}+w_{y, x}^{B}}$

The increasing number of images provides minimum values of the edge preservation measure in the traditional methods of CP-LS and MI [22, 24]. But, the proposed AT achieves maximum edge preservation values for more number of images. Increasing the number of pixels effectively maximizes the $Q A B / F$ of fused image and it leads to fast implementation of segmentation of multi-temporal images. Table 2 describes the comparative analysis of proposed method with the existing methods for $\mathrm{QAB} / \mathrm{F}$.

\begin{tabular}{|l|l|l|l|l|}
\hline \multirow{2}{*}{$\begin{array}{l}\text { Image } \\
\text { No. }\end{array}$} & \multicolumn{4}{|c|}{ QAB/F } \\
\cline { 2 - 5 } & $\begin{array}{l}\text { Joint } \\
\text { Approach }\end{array}$ & CP-LS & MI & $\begin{array}{l}\text { Affine } \\
\text { Transformation }\end{array}$ \\
\hline 1 & 0.632 & 0.592 & 0.615 & 0.735 \\
\hline 2 & 0.658 & 0.618 & 0.659 & 0.754 \\
\hline 3 & 0.542 & 0.502 & 0.523 & 0.716 \\
\hline 4 & 0.563 & 0.52 & 0.544 & 0.728 \\
\hline
\end{tabular}

Table 2: QAB/F Vs Image.

The performance comparison of AT with the existing joint approach, CP-LS and MI models shown in Fig. 8 based on the parameter of $\mathrm{QAB} / \mathrm{F}$. The highest value of $\mathrm{QAB} / \mathrm{F}$ for the $\mathrm{AT}$ is 0.754 . The performance value of $\mathrm{QAB} / \mathrm{F}$ is increased and so the proposed method produces the improved edge preservation value. The value of $\mathrm{QAB} / \mathrm{F}$ is a distance metric.

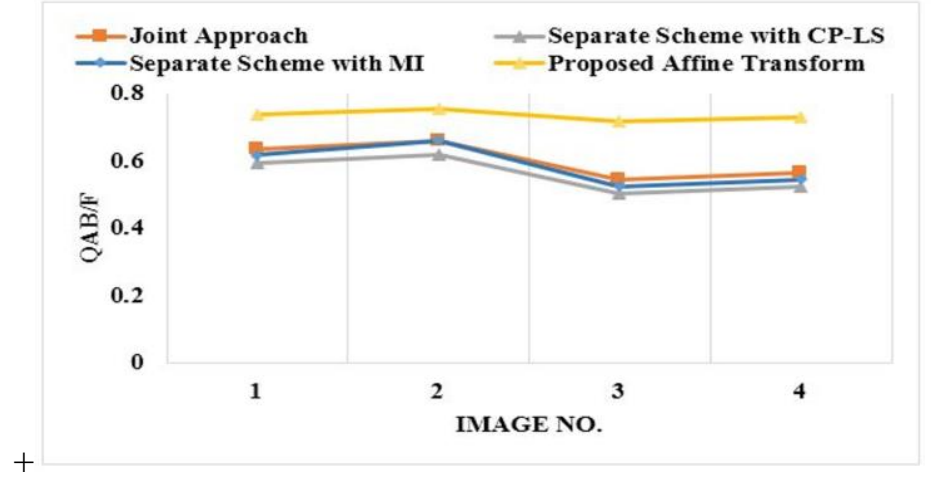

Figure 8: QAB/F vs. Image No 


\subsection{Mutual Information}

The sum of the MIs between the individual images and the fused image is the criterion to describe the performance of the proposed technique. The image fusion performance $(I)$ between input images $(A, B)$ to $F$ with pixels $(p)$ is given by

$I_{F A}(f, a)=\sum_{f, a} p_{F A}(f, a) \log \frac{p_{F A}(f, a)}{p_{F}(f) p_{A}(a)}$

$I_{F B}(f, b)=\sum_{f, b} p_{F B}(f, b) \log \frac{p_{F B}(f, b)}{p_{F}(f) p_{B}(b)}$

The MI between the input images $\left(M I_{F}^{A B}\right)$ and $F$ is given by

$M I_{F}^{A B}=I_{F A}(f, a)+I_{F B}(f, b)$

The increasing number of images provide the minimum values for the MI between the images and fused image in the traditional methods of MFIF and DWT but, it provides maximum in proposed PSO-AT method. Increasing the number of pixels effectively maximizes the MI of fused image leads to better fusion result. Table 3 describes the comparative analysis of proposed method with the existing methods for MI.

\begin{tabular}{|l|l|l|l|}
\hline $\begin{array}{l}\text { Image } \\
\text { No. }\end{array}$ & \multicolumn{3}{|c|}{ MI } \\
\cline { 2 - 4 } & DWT & MFIF & $\begin{array}{l}\text { Affine } \\
\text { Transformation }\end{array}$ \\
\hline 1 & 7.47 & 9.63 & 10.79 \\
\hline 2 & 7.73 & 9.16 & 10.59 \\
\hline 3 & 8.54 & 11.54 & 12.76 \\
\hline 4 & 7.29 & 9.89 & 11.49 \\
\hline
\end{tabular}

Table 3: MI Vs Image No.

The performance comparison of affine transformation with the existing joint approach, MFIF and DWT models are shown in Fig. 9 based on the parameter of MI. The highest value of MI for the proposed PSO-AT is 12.76. Since the performance value of MI is increased, the proposed method produces improved fusion performance. The value of MI is a distance metric.

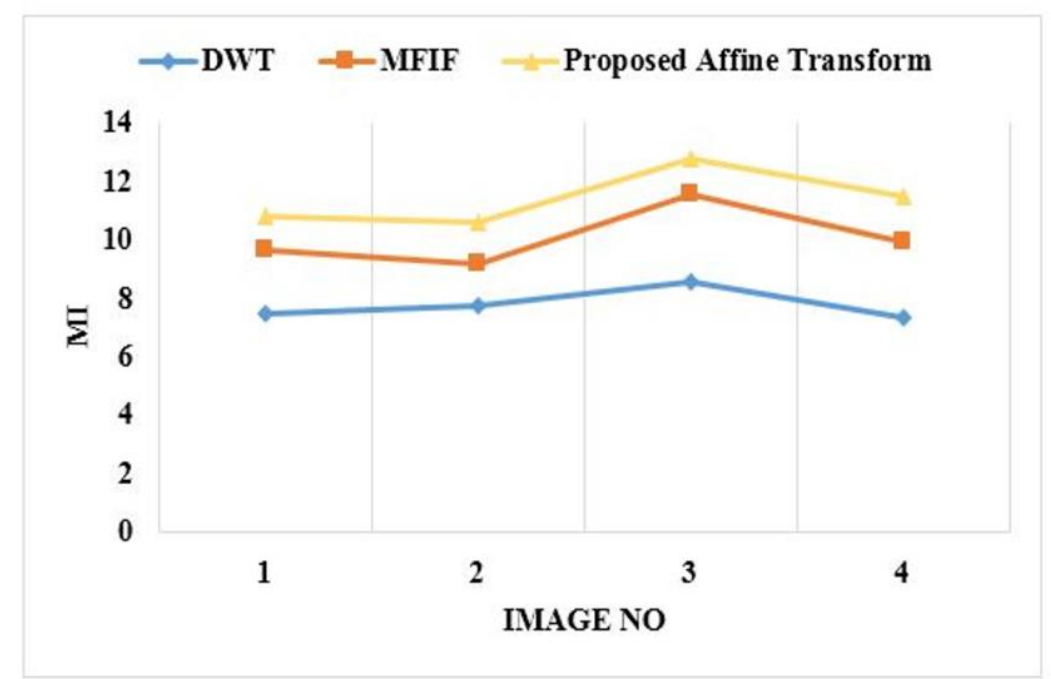

Figure 9: MI vs. Image No. 


\section{Conclusion}

In this paper, affine transformation for efficient cloud removal and enhanced image registration process in multi-temporal image processing is proposed. In affine transformation, the PSO based feature points for the reference images and the new images are taken as the inputs and they are transformed to affine space. Initially, the PSO based feature extraction on twelve angle variation performed and selected the best location affine transformation space. The particle position and velocity updating with respect to changes in the objective function calculation. The suitable initial guess in objective function based on image center and the nonmovement of an image in affine space offered the considerable reduction in time and complexity and the improvement in edge preservation measure. The proposed technique analyzed the parameters of computation time against number of pixels of an image with the traditional methods like CRS and MRF. Also, the QAB/F and the MI between the pixels for the number of images are obtained. The comparative analysis of the performance parameters with the traditional methods of CP-LS, MFIF and DWT models confirmed the effectiveness of the proposed system. The results confirmed the proposed PSO based affine transformation for registration process deviation of $\mathrm{MI}$ value to $25.8 \%$ and $\mathrm{QAB} / \mathrm{F}$ to $12.3 \%$ than the existing methods.

\section{References}

[1] S. Y. Kim, D. Cha, S.W. Kim, "Detection of group of stationary targets using high-resolution SAR and EO images", Proc. of the 2014 IEEE International Geoscience and Remote Sensing Symposium, (IGARSS 2014), IEEE, pp. 1769-1772, 2014. DOI: 10.1109/IGARSS.2014.6946795

[2] T. Celik, K. K. Ma, "Multitemporal image change detection using undecimated discrete wavelet transform and active contours", IEEE Transactions on Geoscience and Remote Sensing, 49:706-716, 2011. DOI: $10.1109 /$ TGRS.2010.2066979

[3] M. Gong, Z. Zhou, J. Ma, "Change detection in synthetic aperture radar images based on image fusion and fuzzy clustering", IEEE Transactions on Image Processing, 21:2141-2151, 2012. DOI: 10.1109/TIP.2011.2170702

[4] E. B. Brooks, R. H. Wynne, V. Thomas, C. E. Blinn, J. W. Coulston, "On-the-fly massively multitemporal change detection using statistical quality control charts and Landsat data", IEEE Transactions on Geoscience and Remote Sensing, 52:3316-3332, 2014. DOI: 10.1109/TGRS.2013.2272545

[5] B. Demir, F. Bovolo, L. Bruzzone, "Detection of land-cover transitions in multitemporal remote sensing images with active-learning-based compound classification", IEEE Transactions on Geoscience and Remote Sensing, 50:1930-1941, 2012. DOI: 10.1109/TGRS.2011.2168534

[6] B. Zitová, J. Flusser, "Image registration methods: a survey", Image and Vision Computing, 21: 9771000, 2003. DOI: 10.1016/S0262-8856(03)00137-9

[7] H. J. Johnson, G. E. Christensen, "Consistent landmark and intensity-based image registration”, IEEE Transactions on Medical Imaging, 21:450-461, 2002. DOI: 10.1109/TMI.2002.1009381

[8] J. Kim, J. Fessler, "Intensity-based image registration using robust correlation coefficients", IEEE Transactions on Medical Imaging, 23:1430-1444, 2004. DOi: 10.1109/TMI.2004.835313

[9] A. Averbuch, Y. Keller, "FFT based image registration" in Proc. of the 2002 IEEE International Conference on Acoustics, Speech, and Signal Processing (ICASSP 2002), pp. IV-3608-IV-3611, 2002. DOI: 10.1109/ICASSP.2002.1004697

[10] H. S. Stone, M. T. Orchard, E.C. Chang, S. Martucci, "A fast direct Fourier-based algorithm for subpixel registration of images", IEEE Transactions on Geoscience and Remote Sensing, 39:22352243, 2001. DOI: $10.1109 / 36.957286$ 
[11] G. C. Sharp, S. W. Lee, D. K. Wehe, "ICP registration using invariant features", IEEE Transactions on Pattern Analysis and Machine Intelligence, 24:90-102, 2002. DOI: 10.1109/34.982886

[12] I. Stamos, M. Leordean, "Automated feature-based range registration of urban scenes of large scale", in Proc. of the 2003 Conference on Computer Vision and Pattern Recognition (CVPR 2014), IEEE Computer Society, pp. II-555-Ii-561, vol. 2, 2003. DOI: 10.1109/CVPR.2003.1211516

[13] R. Qin, A. Gruen, "3D change detection at street level using mobile laser scanning point clouds and terrestrial images", ISPRS Journal of Photogrammetry and Remote Sensing, 90:23-35, 2014. DOI:10.1016/j.isprsjprs.2014.01.006

[14] T. Kroeger and L. Van Gool, "Video registration to SfM models", in Proc. of the 2014 European Conference on Computer Vision, ECCV 2014, ed: Springer, pp. 1-16, 2014. DOI: 10.1007/978-3-31910602-1_1

[15] J. Ruttle, C. Arellano, R. Dahyot, "Robust shape from depth images with GR 2 T", Pattern Recognition Letters, 50:43-54, 2014. DOI: 10.1016/j.patrec.2014.01.016

[16] G. Camps-Valls, D. Tuia, L. Bruzzone, J. Atli Benediktsson, "Advances in hyperspectral image classification: Earth monitoring with statistical learning methods", Signal Processing Magazine, IEEE, 31:45-54, 2014. DOI: 10.1109/MSP.2013.2279179

[17] S. B. Serpico, L. Bruzzone, G. Corsini, W. J. Emery, P. Gamba, A. Garzelli, "Development and validation of multitemporal image analysis methodologies for multirisk monitoring of critical structures and infrastructures", in Proc. of the 2012 International Geoscience and Remote Sensing Symposium (IGARSS 2012), IEEE, pp. 5506-5509, 2012.

[18] C. Benedek, X. Descombes, and J. Zerubia, "Building development monitoring in multitemporal remotely sensed image pairs with stochastic birth-death dynamics", IEEE Transactions on Pattern Analysis and Machine Intelligence, 34:33-50, 2012. DOI: 10.1109/TPAMI.2011.94

[19] W. Li and H. Leung, "A maximum likelihood approach for image registration using control point and intensity", IEEE Transactions on Image Processing, 13:1115-1127, 2004. DOI: 10.1109/TIP.2004.828435

[20] S. Klein, M. Staring, K. Murphy, M. Viergever, and J. P. Pluim, "Elastix: a toolbox for intensity-based medical image registration", IEEE Transactions on Medical Imaging, 29:196-205, 2010. DOI: 10.1109/TMI.2009.2035616

[21] T. Vercauteren, X. Pennec, A. Perchant, and N. Ayache, "Non-parametric diffeomorphic image registration with the demons algorithm", Proc. of the 2007 Medical Image Computing and ComputerAssisted Intervention (MICCAI 2007), pp. 319-326, 2007.

[22] S. Li and B. Yang, "Multifocus image fusion using region segmentation and spatial frequency", Image and Vision Computing, 26:971-979, 2008. DOI: 10.1016/j.imavis.2007.10.012

[23] C. Berger, M. Voltersen, R. Eckardt, J. Eberle, T. Heyer, N. Salepci, et al., "Multi-modal and multitemporal data fusion: Outcome of the 2012 GRSS data fusion contest", IEEE Journal of Selected Topics in Applied Earth Observations and Remote Sensing, 6:1324-1340, 2013. DOI: 10.1109/JSTARS.2013.2245860

[24] S. Chen, Q. Guo, H. Leung, and É. Bossé, “A maximum likelihood approach to joint image registration and fusion", IEEE Transactions on Image Processing, 20:1363-1372, 2011. DOI: 10.1109/TIP.2010.2090530

[25] D. Mahapatra and Y. Sun, "Integrating segmentation information for improved MRF-based elastic image registration", IEEE Transactions on Image Processing, 21:170-183, 2012. DOI: 10.1109/TIP.2011.2162738 
[26] AVIRIS image Indian Pine Test Site https://engineering.purdue.edu/ biehl/MultiSpec/hyperspectral.html

[Online].

Available: 